Eine interstitielle Lungenbeteiligung bleibt bei der systemischen Sklerose klinisch oft lange unerkannt. Der Ultraschall bildet Veränderungen mit typischen Schweifartefakten ab. Wie valide und zuverlässig die Methode ist, untersuchte die Arbeitsgruppe OMERACT mit einem systematischen Literaturüberblick.

Mehr als die Hälfte der Patienten mit systemischer Sklerose (systemische Sklerodermie SSc) bekommen eine interstitielle Lungenerkrankung (ILD). Die ILD tritt u.U. bereits in den ersten 4 Krankheitsjahren auf. Die Lungenfunktionsprüfung ist dann möglicherweise noch unauffällig und eine Lungenbiopsie erfolgt nicht. Den bildgebenden Verfahren komme daher eine Schlüsselrolle zu, so die OMERACT-Gruppe (Outcome Measures in Rheumatology/Ultrasound Group). Der Röntgen-Thorax hat für die ILD bei SSc eine geringe Sensitivität. Eine zuverlässige Diagnostik erfolgt mit der hochauflösenden Computertomografie (HRCT), die aber mit einer Strahlenbelastung und hohen Kosten verbunden ist. Der Lungenultraschall (LUS) bietet eine kostengünstige Alternative, deren Validität, Zuverlässigkeit, Machbarkeit und Standardisierung offen waren. Eine ILD zeigt sich im LUS mit B-Linien, die aus Comet-Tail-Artefakten bestehen (Schweifartefakte durch Wiederholungsechos). OMERACT bildete eine internationale Arbeitsgruppe, überprüfte die Rolle des LUS für das Assessment der ILD/SSc und diskutierte die aktuelle Evidenzlage.

12 Publikationen aus den Jahren 19972017 wurden in die Analyse aufgenommen. Dabei handelte es sich um Beobachtungs-, Querschnitts- und deskriptive Studien. Randomisierte Untersuchungen standen nicht zur Verfügung. Insgesamt lagen Daten von 635 Patienten mit einer SSc vor (median 36,5 Personen/Studie). Die Krankheitsdauer betrug median 5,3 Jahre und $82 \%$ waren Frauen. Typischerweise trat die SSc in der 6. Lebensdekade auf. Der Subtyp war überwiegend nicht bekannt.

\section{Systemische Sklerose im thorakalen Ultraschall}

Gutierrez M et al. Ultrasound in the Assessment of Interstitial Lung Disease in Systemic Sclerosis: A Systematic Literature Review by the OMERACT Ultrasound Group. J Rheumatol 2020; 47: 991-1000. doi:10.10.3899/jrheum.180940
Untersuchungsschema sah die Evaluation der Interkostalräume vor. Die anteriore und laterale Begutachtung erfolgte in Rückenlage. Posteriore und dorsale Lungenabschnitte wurden sitzend untersucht. Die LUS fand mit unterschiedlichen Ultraschallköpfen statt (Linar-, Konvex- und Sektorsonden). Generell wurden für die linearen Transducer 8-11 MHz und für die konvexen 3,5-5 MHz benutzt. Nur in 3 Studien kannte der Untersucher die klinischen Befunde nicht.

Bei $92 \%$ der Studien bestand ein geringes Risiko für eine Datenverzerrung. In der Klassifikation nach den PRISMA-Leitlinien bestand ein Evidenzgrad 2b. In 11 Fällen war die HRCT Komparator als Goldstandard. 4 Untersuchungen nahmen die Spirometrie hinzu. Alle Studien zeigten eine positive Korrelation von LUS-B-Linien und HRCT. Eine multivariate Analyse bestätigte den Zusammenhang jedoch nicht. Für die Bewertung der Zuverlässigkeit und Reproduzierbarkeit lagen unzureichende Daten vor. Lediglich 3 Studien ermittelten $\kappa$-Werte als Maß für die Inter- und Intrareader-Übereinstimmung. Der prognostische Wert des LUS wurde nicht überprüft. Abhängig von der Krankheitsschwere und der verwendeten Technik dauerten die LUS 6-31 min. Informationen über die LUS-Verfügbarkeit und Kosten-Nutzen-Relation fanden sich nicht.

\section{FAZIT}

Die Ergebnisse für den LUS bei SSc seien ermutigend, so die Autoren. Für die Validierung bliebe aber in Folgestudien noch viel zu tun. Hier nennt die Arbeitsgruppe 5 Punkte: übereinstimmende Definitionen für die Auswertung, ein Protokoll für die Datenakquisition, Festlegung der Quantifizierung, übereinstimmende Scoring-Systeme sowie prospektive und langfristige Verlaufsbeobachtungen. Die Gruppe habe kürzlich eine Studie mit 133 SSc-Patienten initiiert, die den diagnostischen Wert bei subklinischen ILD überprüfte. Verglichen mit gesunden Kontrollen wiesen $40,6 \%$ der Erkrankten typische LUS-Zeichen für eine ILD auf.

Dr. Susanne Krome, Melle 